\title{
ON DIRICHLET CONVOLUTION METHOD
}

\author{
INDULATA SUKLA \\ Department of Mathematics \\ Sambalpur University \\ Jyoti Vihar, Sambalpur \\ Orissa, INDIA
}

(Received February 8, 1991 and in revised form April 20, 1995)

\begin{abstract}
In this paper we have proved limitation theorem for $(D, h(n))$ summability methods and have shown that it is best possible.
\end{abstract}

KEY WORDS AND PHRASES: Summability, Dirichlet convolution methods 1991 AMS SUBJECT CLASSIFICATION CODES: $11,11 \mathrm{~N}$

\section{INTRODUCTION}

In his studies on the prime number theorem, Ingham [1] defined a novel summability method called (I) This was generalized by Segal [2] and he defined the notion of $(D, h(n))$ summability, where $h: N \rightarrow R$ denotes a function with $h(1)=1$ We define the "Dirichlet inverse" $h^{*}(n)$ of $h(n)$ by $\sum_{d \mid n}$ $h(d) h^{*}(n / d)=\left\{\begin{array}{l}1, n=1 \\ 0, n>2\end{array}\right.$. A series $\sum a_{n}$ is said to be $(D, h(n))$ summable to $L$ if and only if

$$
n \stackrel{\lim }{\rightarrow} \infty \frac{1}{n} \sum_{v=1}^{n} v \sum_{d \mid v} a_{d} h(v / d)=L .
$$

Given a series $\sum a_{n}$ and a specific $h(n)$, define the function

$$
D(t)=\frac{1}{t} \sum_{n<t} n \sum_{d \mid n} a_{d} h(n / d) .
$$

Since $D([t])=\frac{t}{[t]} D(t)$, it clearly makes no difference to the existence or value of the limit (1 2) whether $t \rightarrow \infty$ is through real values or integers. Ingham's method corresponds to the case $h(n)=\frac{1}{n}$

Segal [3] proved the limitation theorem for (I) summability. If $\sum a_{n}$ is (I) summable, then $\sum_{n<x} a_{n}=o(\log x)$ and has shown in the following theorem that his result is best possible

THEOREM A [4] Let $\in(x)$ be any positive function decreasing to 0 monotonically but arbitrarily slowly as $x \rightarrow \infty$. Then there exists a series $\sum a_{n}$ which is (I) summable and such that

$$
\sum_{n<x} a_{n} \neq 0(\in(x) \log x) \text { as } x \rightarrow \infty .
$$

Sukla [5] has shown an analogous limitation theorem for $(D, h(n))$ summability.

THEOREM B. If $\sum a_{n}$ is $(D, h(n))$ summable then $\sum_{n<x} a_{n}=O(\log x)$ if

and

$$
H^{*}(r)=\sum_{n<r} h^{*}(n)=O(1)
$$

$$
\sum_{v=1}^{n}\left|h^{*}(v)\right|=O(\log n) .
$$


It is remarked in that paper that the condition (ii) cannot be dropped However if we replace (i) by a slightly stronger condition then we get the result to be true without assuming (ii) In section 4 we show that our revised version of Theorem $A$ is best possible.

\section{MAIN RESULTS}

THEOREM 1. If $\sum a_{n}$ is $(D, h(n))$ summable then

if

$$
\sum_{n \leq x} a_{n}=O\left(\sum_{n<x}\left|h^{*}(n)\right|\right)
$$

$$
\sum_{n \leq r} h^{*}(n)=O\left((\log r)^{-1-\epsilon}\right) \quad \text { as } \quad r \rightarrow \infty .
$$

We will show that $(31)$ is a best possible result

THEOREM 2. Let $\in(x)$ be any positive function decreasing to 0 monotonically but arbitrarily slowly as $x \rightarrow \infty$. Then there exists a series $\sum a_{n}$ which is $(D, h(n))$ summable and (32) holds and

$$
\sum_{1 \leq d \leq \frac{r}{\left[r^{1 / 2}\right]+1}}\left|h^{*}(d)\right| / \sum_{n \leq \tau}\left|h^{*}(n)\right|
$$

does not tend to zero as $r \rightarrow \infty$ holds and such that

$$
\sum_{n \leq x} a_{n} \neq o\left(\epsilon(x) \sum_{n \leq x}\left|h^{*}(n)\right|\right) \text { as } x \rightarrow \infty .
$$

PROOF OF THEOREM 1. For $m \geq 0$, let

$$
K(m)= \begin{cases}m D(m) & \text { if } m \geq 1 \\ 0 & \text { if } m=0\end{cases}
$$

then by (1 1) and (1.2) it follows that

$$
\begin{gathered}
K(m)=O(m), \quad \text { as } n \rightarrow \infty, \quad \text { and } \\
\sum_{n \leq r} a_{n}=\sum_{d \leq r} \frac{K(d)}{d} d\left(\frac{H^{*}\left(\frac{r}{d}\right)}{d}-\frac{H^{*}\left(\frac{r}{d+1}\right)}{d+1}\right) .
\end{gathered}
$$

By (2 4) it is enough to show that

$$
\sum_{d \leq r} d\left|\frac{H^{*}\left(\frac{r}{d}\right)}{d}-\frac{H^{*}\left(\frac{r}{d+1}\right)}{d+1}\right|=O\left(\sum_{n \leq r}\left|h^{*}(n)\right|\right) .
$$

The left hand side of (2.5) is maximized by

$$
\sum_{d \leq r}\left|H^{*}\left(\frac{r}{d}\right)-H^{*}\left(\frac{r}{d+1}\right)\right|+\sum_{d \leq r} \frac{\left|H^{*}\left(\frac{r}{d+1}\right)\right|}{d+1}
$$

Now

$$
\sum_{d \leq r}\left|H^{*}\left(\frac{r}{d}\right)\right|-H^{*}\left(\frac{r}{d+1}\right)=\sum_{d \leq r}\left|\sum_{\frac{r}{d+1} \leq v \leq \frac{r}{d}} h^{*}(v)\right| \leq \sum_{1 \leq v \leq r}\left|h^{*}(v)\right|
$$

and

$$
\sum_{d \leq r}\left|\frac{H^{*}\left(\frac{r}{d+1}\right)}{d+1}\right|=O\left(\sum_{d \leq r-2}\left(\log \frac{r}{d+1}\right)^{-1-\epsilon} \frac{1}{d+1}\right)=O(1)
$$

since $H^{*}(x)=O$ for $x<1$

PROOF OF THEOREM 2. Define $b_{n}$ by 


$$
b_{n}=\sum_{d ! n} h^{*}\left(\frac{n}{d}\right)\left(\frac{d D(d)-(d-1) D(d-1)}{d}\right), \text { where } \quad D(t) \rightarrow 0 \quad \text { as } \quad t \rightarrow \infty
$$

then

$$
\frac{1}{t} \sum_{n<t} n \sum_{d \mid n} b_{d} h\left(\frac{n}{d}\right)=\frac{1}{t} \sum_{n<t} n \sum_{d \mid n} h\left(\frac{n}{d}\right) \sum_{r \mid d} h^{*}\left(\frac{r}{d}\right)\left(\frac{r D(r)-(r-1) D(r-1)}{r}\right)=D(t) .
$$

Since $D(t) \rightarrow 0, \sum b_{n}$ is $(D, h(n))$ summable to 0 .

$$
\begin{aligned}
\sum_{n \leq r} b_{n} & =\sum_{n<r} \sum_{d \mid n} h^{*}\left(\frac{n}{d}\right)\left(\frac{d D(d)-(d-1) D(d-1)}{d}\right) \\
& =\sum_{d \leq r}\left(\frac{d D(d)-(d-1) D(d-1)}{d}\right) \sum_{m \leq r} h^{*}(m) \\
& =\sum_{d \leq r} D(d)-D(d-1) H^{*}\left(\frac{r}{d}\right)+\sum_{d \leq r} \frac{D(d-1)}{d} H^{*}\left(\frac{r}{d}\right)=\sum_{1}+\sum_{2}
\end{aligned}
$$

Now

$$
\sum_{1}=\sum_{d \leq r} D(d)\left[H^{*}\left(\frac{r}{d}\right)-H^{*}\left(\frac{r}{d+1}\right)\right]
$$

Since $H^{*}(x)=0$ for $x<1$

$$
\sum_{2}=O\left(\sum_{d \leq r} \frac{1}{d}\left|H^{*}\left(\frac{r}{d}\right)\right|\right)=O(1) \quad \text { as } \quad \mathrm{r} \rightarrow \infty \quad \text { by }(2.2) .
$$

We have now

$$
\sum_{1}=\sum_{n \leq r} b_{n}+O(1)
$$

Suppose the theorem does not hold then

$$
\sum_{n \leq r} b_{n}=o\left(\epsilon(r) \sum_{n \leq r}\left|h^{*}(n)\right|\right) .
$$

So (2 9) becomes

$$
\sum_{1}=o\left(\epsilon(r) \sum_{n \leq r}\left|h^{*}(n)\right|\right)
$$

Since $D(d) \rightarrow 0$ as $n \rightarrow \infty$, let

$$
\alpha_{r, d}=\frac{1}{\epsilon(r) \sum_{n \leq r}\left|h^{*}(n)\right|}\left[H^{*}\left(\frac{r}{d}\right)-H^{*}\left(\frac{r}{d+1}\right)\right] .
$$

It is well known that in order for $\alpha_{r, d}$ to transform all sequences tending to 0 into sequences tending to 0 ,

$$
\frac{1}{\epsilon(r) \sum_{n \leq r}\left|h^{*}(n)\right|} \sum_{d \leq r}\left|H^{*}\left(\frac{r}{d}\right)-H^{*}\left(\frac{r}{d+1}\right)\right|<c
$$

must hold for all $r$ where $c$ is independent of $r$

$$
\sum_{d \leq r}\left|H^{*}\left(\frac{r}{d}\right)-H^{*}\left(\frac{r}{d+1}\right)\right|=\sum_{d \leq r}\left|\sum_{\frac{r}{d+1}<m \leq \frac{r}{d}} h^{*}(m)\right| \geq \sum_{r^{1 / 2}<d \leq r}\left|\sum_{\frac{r}{d+1}<m \leq \frac{r}{d}} h^{*}(m)\right| .
$$


Since in this last sum $\frac{r}{d}-\frac{r}{d+1}<1$ the inner sum contains at most one term, and so

$$
\frac{1}{\epsilon(r) \sum_{n \leq r}\left|h^{*}(n)\right|} \sum_{d \leq r}\left|H^{*}\left(\frac{r}{d}\right)-H^{*}\left(\frac{r}{d+1}\right)\right| \geq \frac{1}{\epsilon(r)}\left(\frac{\sum_{1<d \leq \frac{1}{|r / 2|+i}}\left|h^{*}(d)\right|}{\sum_{n \leq r}\left|h^{*}(n)\right|}\right)
$$

tends to infinity as $r \rightarrow \infty$ since by (2.3) the expression in the bracket does not tend to zero as $r \rightarrow \infty$ This completes the proof of Theorem 2

Agnew [6] showed directly that, for $r>0$ the Cesáro and Riesz transforms $C_{r}(n), R_{r}(n)$ respectively of a given series $\sum a_{n}$ are equiconvergent i.e $C_{r}(n), R_{r}(n)$ exist for each $n$ and

$$
r \stackrel{\lim }{\rightarrow} \infty\left(C_{r}(n)-R_{r}(n)\right)=0 .
$$

These concepts are applied to arithmetic summation methods (I) and $(D, h(n))$ for particular values of $h(n)$ by Jukes [7] He has found different conditions under which the equiconvergence of $\frac{6}{\pi^{2}}(I)$ and $\left(D, \frac{\mu^{2}(n)}{n}\right)$ have been established. The $\left(D, \frac{\mu^{2}(n)}{n}\right)$ and $\frac{6}{\pi^{2}}(I)$ transform are given by

$$
b_{n k}=\frac{k}{n} \sum_{r \leq \frac{n}{k}} \mu^{2}(r), \quad C_{n k}=\frac{6}{\pi^{2}} \frac{k}{n}\left[\frac{n}{k}\right]
$$

respectively Let $M_{2}=\lim _{n} \sup \sum k\left|\triangle\left(\frac{b_{n k}-c_{n k}}{k}\right)\right|$

$$
A_{2}=\lim _{n} \sup \left|\sum_{k=\infty}^{n} \frac{k a_{k}}{(n+1)}\right| .
$$

THEOREM C [7] Tauherian constants $M_{2}$ do not exist for comparisons of conservative matrices with non-conservative matrices

THEOREM D [7] The $\left(D, \frac{\mu^{2}(n)}{n}\right)$ and $6 / \pi^{2}(I)$ transform are not equiconvergent whenever $A_{2}<\infty$

We have proved (see Kuttner and Sukla [8]) that

THEOREM E. The $(D, h(n))$ is conservative if and only if $\sum_{n=1}^{\infty}|h(n)|<\infty \quad$ It is to note that if part of the above theorem was proved earlier by Jukes [9] See S. $\stackrel{n=1}{\mathrm{~L}}$. Segal, Math. Reviews $86 \mathrm{e} 11093$ (May 1986, p 1864)

THEOREM 3. The $(D, h(n))$ and (I) are not equiconvergent whenever $A_{2}<\infty$ and $\sum|h(n)|<\infty$

PROOF. By Theorem $\mathrm{C}$ since (I) is not conservative and $(D, h(n))$ is conservative for $\left.\sum \mid h(n)\right) \mid<\infty$ whenever $A_{2}<\infty(D, h(n))$ and (I) are not equiconvergent

From Theorem $\mathrm{E}$ also we get that the following theorem of Jukes as corollaries

COROLLARY 1. The methods $\left(D, \frac{\mu(n)}{n}\right)$ and $\left(D, \frac{\lambda(n)}{n}\right)$ are not conservative

PROOF. Since $\sum_{n=1}^{\infty} \frac{\mu(n)}{n}$ and $\sum_{n=1}^{\infty} \frac{\lambda(n)}{n}$ are not absolutely convergent So by Theorem 3 the result follows.

COROLLARY 2. $\left(D, \mu^{2}(n) / n\right)$ and $\left(D, \in \lambda(n) / \pi^{2}(n)\right)$ transforms are not equiconvergent whenever $A_{2}<\infty$.

ACKNOWLEDGMENT. We are thankful to the referee for his valuable suggestions for the improvement of the paper 


\section{REFERENCES}

[1] INGHAM, A E , Some Tauberian theorems connected with prime number theorem, J. of London Math. Soc. 20 (1945), 171-80.

[2] SEGAL, S L , Summability by Dirichlet convolution roc, Camb. Phil. Soc. 63 (1967), 393-400

[3] SEGAL, S.L, On the Ingham summation methods, Canadian J. of Math. 18 (1966), 97-105

[4] SEGAL, S L., A second note on Ingham's summation methods, Canadian Math. Bulletin 22 (1) (1979), $117-20$

[5] SUKLA, I.L., Limitation theorem for $(D, h(n))$ summability, J. of Indian Math. Soc. 48 (1984), 101-105

[6] ANGEW, R.P , Equiconvergence of Cesare and Riesz transforms of series, Duke Math. J. 22 (1955), 451-60

[7] JUKES, K.A, Equiconvergence of matrix transformation, Proc. Amerl. Math. Soc. 69 (2) (1978), 261-70

[8] KUTTNER, B and SUKLA, I.L, On $(D, h(n))$ summability methods, Proc. Camb. Phil. Soc. 97 (1985), 189-93

[9] JUKES, K A, On the Ingham and $(D, h(n))$ summation methods, J. of London Math. Soc. (R) 3 (1971), 699-710 


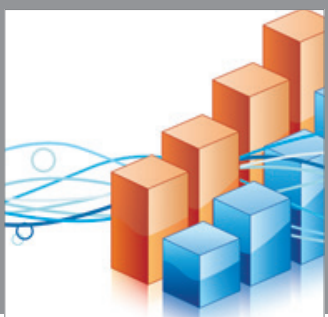

Advances in

Operations Research

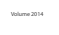

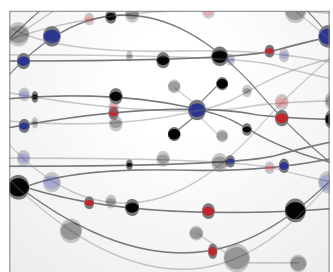

\section{The Scientific} World Journal
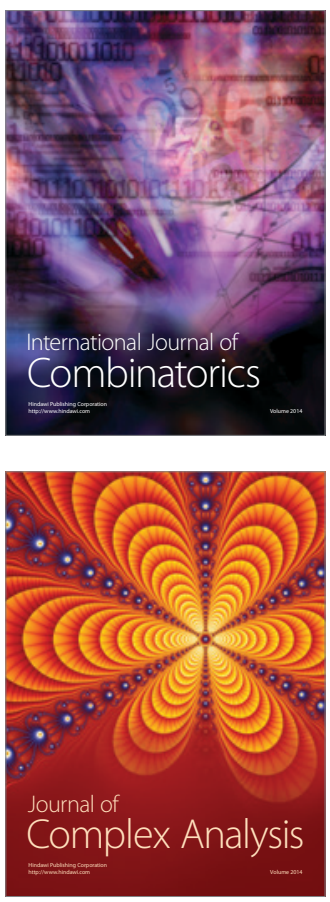

International Journal of

Mathematics and

Mathematical

Sciences
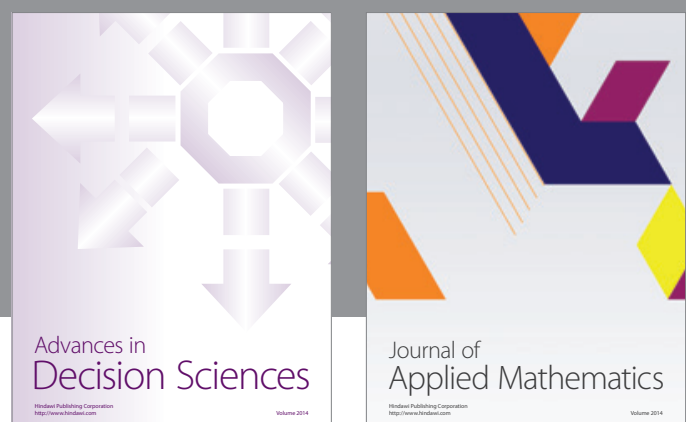

Journal of

Applied Mathematics
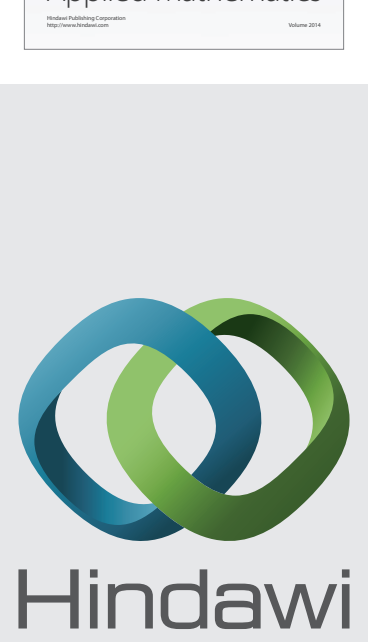

Submit your manuscripts at http://www.hindawi.com
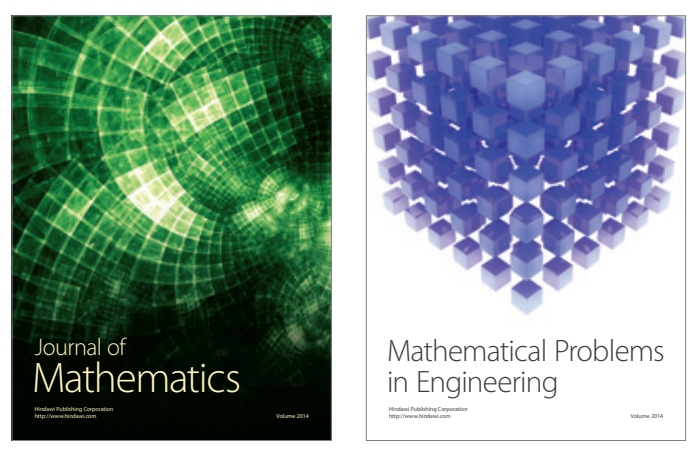

Mathematical Problems in Engineering
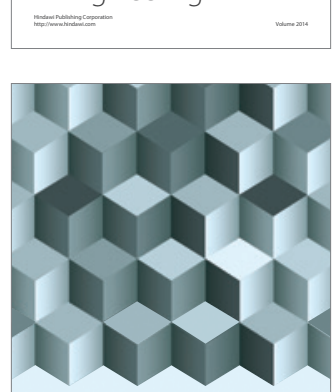

Journal of

Function Spaces
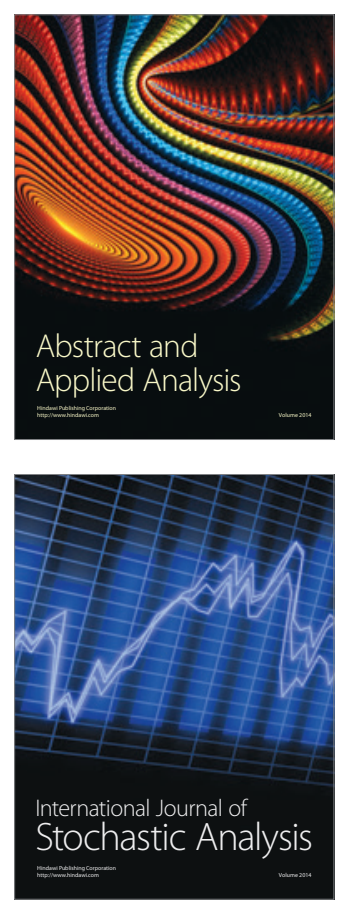

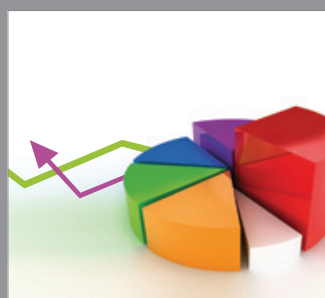

ournal of

Probability and Statistics

Promensencen
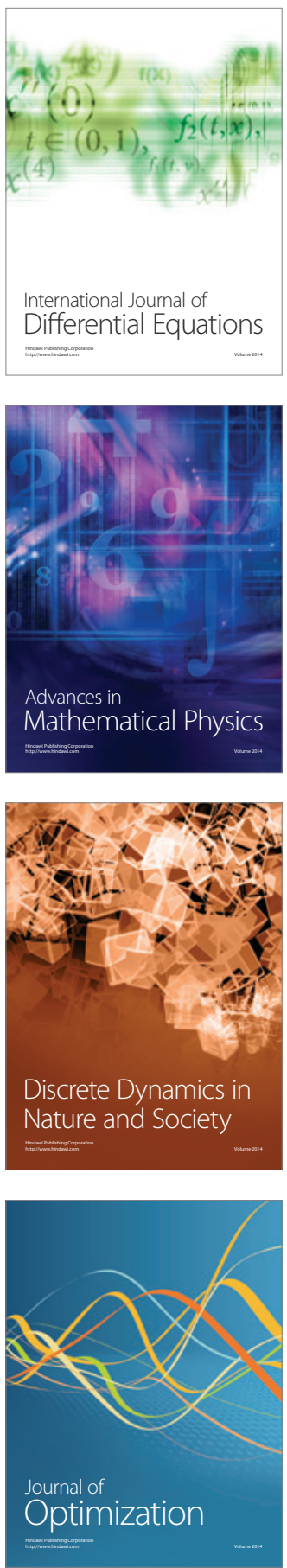